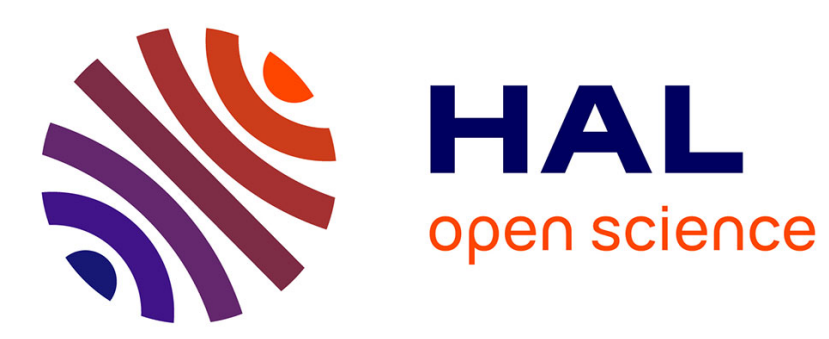

\title{
L'inaltérabilité des géminées et la spirantisation
}

\author{
Rachid Ridouane
}

\section{To cite this version:}

Rachid Ridouane. L'inaltérabilité des géminées et la spirantisation. Études et documents berbères, 2008, 27, pp.127-149. halshs-00384930

\section{HAL Id: halshs-00384930 \\ https://shs.hal.science/halshs-00384930}

Submitted on 17 May 2009

HAL is a multi-disciplinary open access archive for the deposit and dissemination of scientific research documents, whether they are published or not. The documents may come from teaching and research institutions in France or abroad, or from public or private research centers.
L'archive ouverte pluridisciplinaire HAL, est destinée au dépôt et à la diffusion de documents scientifiques de niveau recherche, publiés ou non, émanant des établissements d'enseignement et de recherche français ou étrangers, des laboratoires publics ou privés. 


\title{
L'inaltérabilité des géminées et la spirantisation
}

\author{
Rachid Ridouane \\ Laboratoire de Phonétique et Phonologie (UMR 7018) \\ CNRS/Sorbonne Nouvelle, 19, rue des Bernardins - 75005 Paris \\ Tél.: ++33 (0)1 44320570 - Fax: ++33 (0)1 44320573 \\ rachid.ridouane@wanadoo.fr
}

Cet article traite du comportement des géminées vis-à-vis de la spirantisation en berbère chleuh de Haha $(\mathrm{PCH})$ et de la façon dont le cadre de la Phonologie CV rend compte de ce comportement. La spirantisation est un processus phonologique par lequel certaines occlusives simples (1a) se réalisent comme des fricatives correspondantes. Leurs contreparties géminées (1b) résistent à l'application de cette règle.

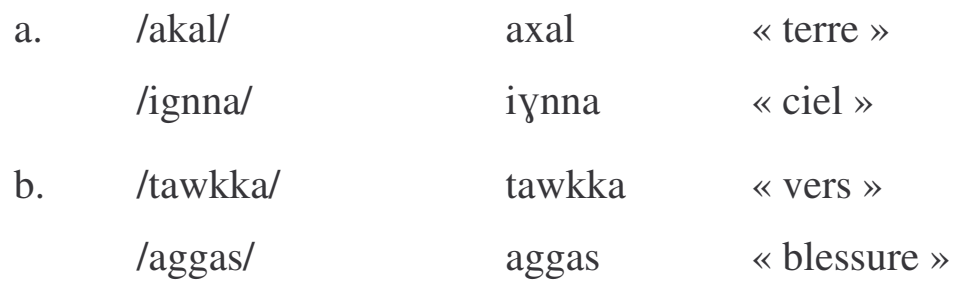

L'incapacité des géminées à se spirantiser est un aspect universel qui reflète «l'inaltérabilité » de ces segments (Hayes 1986, Schein et Steriade 1986, Churma 1988, Kirchner 2000). Différentes analyses ont été avancées pour rendre compte de ce comportement particulier.

\section{Sur l'inaltérabilité des géminées}

\section{1. le traitement notationnel de l'inaltérabilité}

Hayes (1986), à qui l'on doit la notion d' « inaltérabilité » des géminées, a été un des premiers phonologues à avoir traité de cette question de manière systématique et approfondie. Il a analysé, entre autres, des données tirées du tigrinya, dont quelques exemples sont reproduits en (2).

\footnotetext{
${ }^{1}$ Les analyses présentées dans cette section rentrent dans le cadre de ce qu'Elmedlaoui (1993) appelle : « Notational Treatments of geminates ». Nous lui empruntons cette phrase pour servir de titre de cette section. D'autres analyses qui ne rentrent pas dans ce cadre ont été récemment développées. Nous présenterons notamment les résultats de l'analyse d'Elmedloui (1993) pour le cas du berbère dans la section suivante.
} 


\begin{tabular}{|c|c|c|c|}
\hline \multirow{3}{*}{ a. } & Singulier & Pluriel & \\
\hline & $\mathrm{k} \Lambda \mathrm{lbi}$ & Paxalib & « chien $»$ \\
\hline & $\mathrm{g} \Lambda \mathrm{n} \mathrm{Pi}$ & Payani主? & $\ll$ terrain $»$ \\
\hline b. & $\mathrm{f} \Lambda \mathrm{kk} \Lambda \mathrm{r} \Lambda$ & $\mathrm{f} \Lambda \mathrm{kk} \Lambda \mathrm{r} \Lambda$ & «il s'est vanté » \\
\hline c. & mìrak-ka & mìrax-ka & « veau, 2sg. masc. » \\
\hline$d$ & $y \dot{z}-t-k \Lambda f \Lambda t$ & $\mathrm{yizk} \wedge \mathrm{f} \wedge \mathrm{t}$ & « ouvrir, passive» \\
\hline
\end{tabular}

Dans cette langue, à l'instar de l'hébreu biblique (Barkai 1974)², les occlusives postvocaliques se spirantisent (2a). Par contre, la spirantisation n'affecte pas la géminée (2b) même si la première moitié de ce segment est post-vocalique. Hayes postule la règle suivante pour rendre compte de ces données :

(3) La spirantisation en tigrinya :

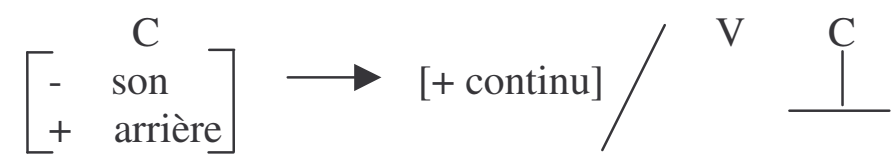

Selon lui, il est possible de prédire les règles phonologiques qui ne peuvent pas affecter les géminées : les règles qui mentionnent dans leurs descriptions structurelles la couche mélodique ET la couche prosodique. Les règles qui échappent à l'inaltérabilité ne mentionnent qu'une seule de ces couches. Voici les critères établis par Hayes pour déterminer dans quel cas une règle doit mentionner une couche particulière :

1. Toute règle qui mentionne les traits distinctifs autres que le trait [syllabique] doit mentionner la couche mélodique

2. Toute règle qui fait appel à une classe de consonnes ou une classe de voyelles doit mentionner la couche prosodique où la syllabicité est représentée

3. Toute règle qui fait référence à la position au sein de la syllabe doit mentionner la couche prosodique.

Un aspect important caractérisant les règles qui mentionnent les deux couches est qu'elles incluent les lignes d'association dans leurs descriptions structurelles. Pour rendre compte de cet aspect, Hayes postule la contrainte suivante :

\footnotetext{
${ }^{2}$ En hébreu biblique, la spirantisation affecte toutes les occlusives non-emphatiques. En tigrinya, seules les occlusives vélaires se spirantisent.
} 
Linking Constraint :

Association lines in structural descriptions are interpreted as exhaustive.

Regardons à présent comment Hayes rend compte des données (2). La règle de spirantisation en tigrinya (4) mentionne les deux couches : la couche prosodique pour identifier la position post-vocalique et la couche mélodique pour identifier l'occlusive vélaire. Cette règle ne pourra pas s'appliquer aux géminées car la description structurelle de cette règle fait référence à une seule ligne d'association entre la cible et son unité prosodique, la géminée étant associée à deux unités prosodiques. Comme l'illustre les représentations en (5), la contrainte d'association prédit aussi la spirantisation du premier $/ \mathrm{k} /$ des formes (2b) ainsi que l'inaltérabilité des géminées issues d'une assimilation complète (2d) :

a. Inaltérabilité de /kk/

$$
\begin{array}{ll}
\mathrm{f} \Lambda \mathrm{kk} \Lambda \mathrm{r} \Lambda & * \mathrm{f} \Lambda \mathrm{xx} \Lambda \mathrm{r} \Lambda \\
\mathrm{VCC} & \\
\mathrm{k} &
\end{array}
$$

b. Spirantisation de /k-k/

$$
\underset{\mathrm{k}}{\mathrm{mizak}-\mathrm{ka}}+\underset{\mathrm{k}}{\mathrm{V}}+\underset{\mathrm{x}}{\mathrm{C}} \longrightarrow \underset{\mathrm{k}}{\mathrm{V}}
$$

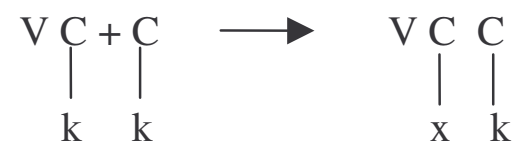

c. Inaltérabilité de /kk/ issu d'assimilation complète :

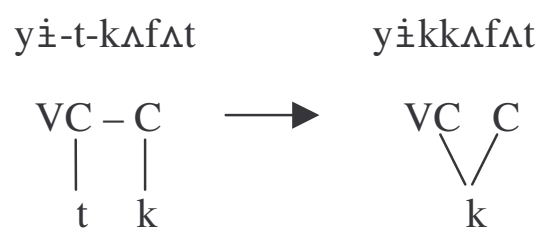

Schein \& Steriade (1986) ont développé une analyse légèrement différente de celle de Hayes. Selon eux, les règles qui s'appliquent aussi bien aux simples qu'aux géminées tendent à appartenir à une même classe de règles qui ne requièrent que des informations segmentales. L'inaltérabilité des géminées est donc entièrement prédictible à partir de la façon dont les règles, qui s'appliquent aux simples, sont formulées : «Rules' applicability to a multiply linked autosegment (in a geminate or in a partially assimilated cluster) can always be predicted from the way the rule must be formulated when only singly linked autosegments are 
considered.» (ibid : 693-4). Ainsi, les structures (6), ci-dessous, peuvent être affectées différemment selon la description structurelle de la règle.

(6)

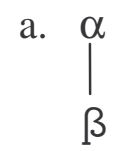

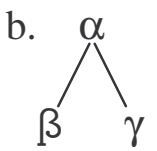

couche A

couche B

Si la règle affectant $\alpha$ impose des conditions qui sont satisfaites par $\beta$ et non par $\gamma$, alors la règle ne pourra pas s'appliquer aux formes ayant la structure (6b). Si par contre, la règle affecte un élément $\alpha$ de la couche A et qu'aucune condition n'est imposée sur les éléments $\beta$ associés à cet $\alpha$, alors la règle s'appliquera aussi bien à (6a) qu'à (6b) sans distinction entre segments simples ou segments doublement liés. Schein \& Steriade établissent la condition suivante pour rendre compte de cet aspect :

\section{Uniform applicability condition :}

Given a node $n$, a set $\mathrm{S}$ consisting of all nodes linked to $n$ on some tier $\mathrm{T}$, and a rule $\mathrm{R}$ that alters the contents of $n$ : a condition in the structural description of $\mathrm{R}$ on any member of $\mathrm{S}$ is a condition on every member of $\mathrm{S}$.

Ils formulent la règle suivante pour décrire le processus de spirantisation post-vocalique des formes $(2 a)$ :

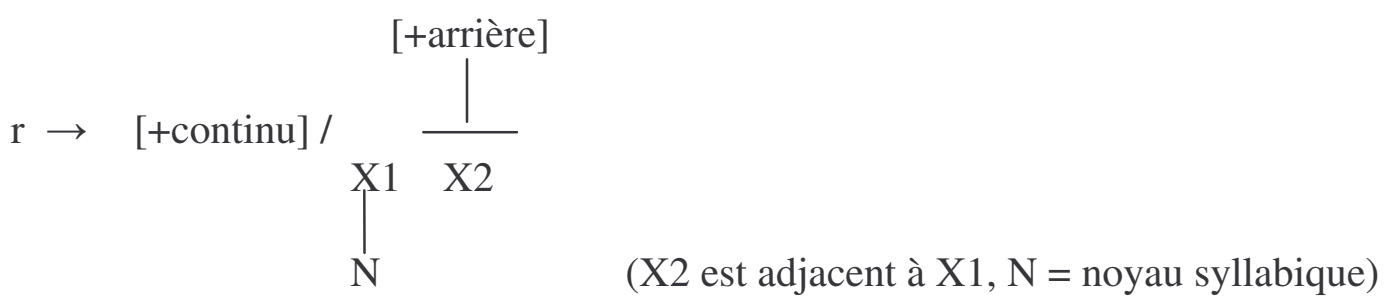

La règle (8) n'est pas une règle segmentale mais plutôt «a dependent structure rule » dans le sens où la règle fait référence aux informations contenues dans la couche mélodique (le trait [+ arrière]) ainsi que dans la couche prosodique (l'adjacence entre le noyau de la syllabe et la cible). Cette adjacence explique le blocage de la spirantisation dans les formes (2b). Des deux positions de la géminée /kk/, seule la première position est adjacente au noyau. Ainsi la règle est bloquée car une seule position $\mathrm{X}$ de la séquence cible satisfait la description structurelle de la règle.

Plusieurs auteurs ont émis des crtiques sérieuses à l'égard ce type d'analyses notationnelles (Churma 1988, Inkelas \& Cho 1993, Elmedlaoui 1993, Kirchner 2000). Selon Elmedlaoui 
(1993 : 133) : «What is common, on the theoretical level, to most of these proposals, and to the move consisting of devising notational phonological constraints in general, is the fact that, [...], those principles and constraints are, so to speak 'established by decree'.» Inkelas \& Cho (1993) ont mis en lumière les lacunes de ces deux analyses en se basant sur des données du coréen. Les processus de syllabification, qui font référence aux informations aussi bien prosodiques que mélodiques devraient, à l'instar des autres processus, faire état d'inaltérabilité due à la contrainte d'association. Pourtant dans cette langue, la règle ou la contrainte qui exclut les vélaires nasales en position d'attaque s'applique aussi bien aux simples qu'aux géminées: [kạ] mais * [ạa, aĐ̣a]. Une autre faiblesse des approches adoptant un traitement notationnel des géminées est qu'elles partagent tacitement, au niveau empirique, le postulat qu'une règle de spirantisation qui s'appliquerait uniquement aux géminées seraient aussi naturelle que celle dont l'application serait limitée aux segments simples (cf. Elmedlaoui 1993). Ces deux types de règles sont concevables au vu de ces propositions et leurs applications ne violeraient aucune des conditions ou contraintes recensées par Hayes et Schein \& Steriade. Une approche différente de celle de Hayes et de Schein \& Steriade a été développée par Elmedlaoui (1993). Cette approche, résumée en bas, prend en considération le lien étroit qui existe, selon Elmedlaoui entre l'inaltérabilité des géminées et les phénomènes de lénition et de fortition.

\subsection{Le 'Principe du développement inertiel' : Elmedlaoui (1993)}

Qu'est-ce que les géminées ont de particulier qui expliquerait leur comportement différent de celui de leurs contreparties simples ? C'est à cette question qu'Elmedlaoui (1993) a essayé d'apporter une réponse satisfaisante en explorant une approche alternative : le Principe de Développement Inertiel (désormais PDI) initié par Foley (1977). La généralisation majeure que le PDI souligne concernant les géminées est que, pour tout ensemble de traits [F] qui participe à la détermination du degré de sonorité des segments, la distribution (sous-jacente ou dérivée) des valeurs $[\alpha \mathrm{F}]$ entre les simples et les géminées est telle que les géminées se voient systématiquement attribuer la valeur $[\alpha \mathrm{F}]$ qui diminue la sonorité, tandis que les segments simples, dans le contexte approprié, se voient systématiquement attribuer la valeur $[-\alpha F]$ qui augmente la sonorité. Ce principe et les généralisations qui en découlent apportent selon Elmedlaoui une réponse à la question suivante: «Pourquoi une alternance simple/géminée comme $b / \beta \beta$ n'est jamais attestée tandis que $\beta / b b$ est très largement répandue ?» 
Il a illustré l'applicabilité de ce principe en examinant l'alternance spirante/occlusive en rifain et en kabyle (en plus de l'hébreu). Selon Elmedlaoui, la direction historique du changement phonétique : occlusive / spirante, ne peut aujourd'hui rendre compte de l'alternance au niveau synchronique. Cette affirmation qui est à la base de son analyse concerne les faits des dialectes berbères suivants tels qu'ils ont été analysés par Saïb (1976) pour Ayt Ndhir, El Kirat (1987) pour le Beni Iznassen, Chaker (1984) pour le kabyle et Tangi (1991) pour le rifain. Selon Elmedlaoui, deux processus historiques ont essentiellement contribué à établir la situation synchronique actuelle :

1. La réduction très importante au point de vue quantitative d'un nombre considérable de géminées lexicales dans des environnements qui ne peuvent plus être «systématisés ».

2. L'emprunt, essentiellement aux dialectes arabes, d'un nombre important de mots contenant des occlusives simples sans les spirantiser.

Le premier changement, l'émergence d'occlusives simples résultant des géminées, a facilité la non spirantisation des occlusives simples des emprunts. Cette nouvelle situation rend donc l'établissement d'une règle synchronique impossible puisque seuls les segments appartenant à un fond lexical ancien participe à la distribution lexicale ou à l'alternance phonologique et morphologique entre les spirantes simples et les géminées occlusives. Pour Elmedlaoui, la seule façon de rendre compte de cette alternance est d'établir une règle d'occlusivication (9) qui pourra rendre compte entre autres des données (10) ci-dessous :

(9) Strengthening Rule (pour le kabyle).

a. [- sonorant $] \longrightarrow[$ - continu $]$

b. $\operatorname{PSPV}^{3}: \mathrm{S}_{\mathrm{N}}$, dans des environnements dérivés.

(Voir plus bas sur la notion $\mathrm{S}_{\mathrm{N}}$ )

$\begin{array}{lll}\underline{\text { Aoriste }} & \underline{\text { Inaccompli }} & \\ \mathrm{f} \theta 1 & \mathrm{fttl} & \text { «rouler» } \\ \chi ð \mathrm{~m} & \chi \mathrm{ddm} & \text { «travailler» } \\ \mathrm{s} \beta \mathrm{R} & \operatorname{sbbR} & \text { «peindre }\end{array}$

La règle d'occlusivication selon Elmedlaoui est un processus de fortition gouverné par le PDI et les généralisations qui en découlent (cf. Foley 1977). Parmi les principes du PDI, les deux ci-dessous ont été légèrement adaptés par Elmedlaoui (ibid. : 140) :

\footnotetext{
${ }^{3}$ PSPV $=$ Positional Strength Parochial Value.
} 
1. Les segments forts et les positions fortes ont priorité dans les processus de fortition de trait et dans la distribution des traits fortis.

2. Les segments faibles et les positions faibles ont priorité dans les processus d'affaiblissement de trait et dans la distribution des traits faibles.

La gémination selon ce principe est placée au bas de l'échelle de sonorité, autrement dit au niveau le plus haut de la force segmentale. Elmedlaoui, contrairement à Foley (1977), ne considère pas la gémination comme une propriété segmentale mais plutôt comme une propriété positionnelle ou moraïque. Par conséquent, il les range, par rapport à leur force positionnelle relative, comme les consonnes les plus fortes, suivies par d'autres positions de plus en plus faibles (ibid. : 145) :

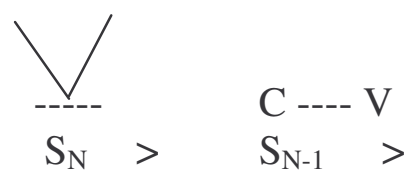

(1)
(2)

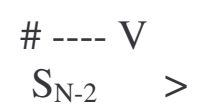

(3)

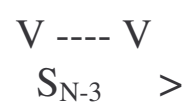

(4)<smiles>[Y][AsH]</smiles>

(5)

Un ensemble de généralisations peut être dérivé du PDI et de la classification (11) :

(12)a. Tout processus de fortition qui affecte un membre de (11) 1-5 affecte d'une façon redondante le reste des membres à sa gauche, et tout processus d'affaiblissement qui affecte un membre de (11) 1-5 affecte d'une façon redondante le reste des membres à sa droite ${ }^{4}$.

b. Aucun processus d'affaiblissement de trait qui affecte un membre ou un ensemble de membres (11) 2 ne peut affecter (11) 1. Autrement dit, les géminées ne subissent jamais directement un processus d'affaiblissement.

c. Dans les systèmes phonémiques où la gémination est réduite à une classe naturelle donnée, cette classe de segments doit être spécifiée exclusivement par les traits de fortition $^{5}$.

La généralisation (12b), celle qui nous intéresse le plus, constitue une manière plus globale et plus drastique d'exprimer la généralisation de Churma (1988: 3) «No spirantization rule is restricted so as to apply only to geminates. » Pour Elmedlaoui, si on assume les principes du PDI et la représentation (11) selon laquelle la structure de la géminée représente la

\footnotetext{
${ }^{4}$ Cette clause constitue un développement de la généralisation de Churma (1988:4) « No stregthening process may affect only simplex consonants »
} 
manifestation la plus forte d'une matrice de traits donnée, on ne peut nullement concevoir une alternance où des géminées peuvent participer à un processus qui introduirait des traits d'affaiblissement [+continu, + approximant, etc.]. Selon cette approche, seuls sont concevables les cas où les segments simples subissent des processus de lénition (weakening) dans un contexte donné tandis que leurs contreparties géminées dans les mêmes contextes, subissent un affaiblissement prosodique consistant en une réduction quantitative, avant de subir éventuellement un processus d'affaiblissement. La «graduation consonantique » en finnois est un exemple de la réduction quantitative (cf. Karlsson (1983 : 30-38) et Selkirk (1990 : 133-136)) et le cas de la dégémination en position finale du mot en hébreu en est un autre ; les deux illustrent le phénomène de la réduction quantitative suivie d'affaiblissement.

Revenons à la règle (9). La clause (9a) stipule, sans plus de détail, que les obstruantes en kabyle se réalisent comme des occlusives. En référence à l'échelle de force positionnelle (11), la clause (9b) considère les géminées comme le point paramétrique de l'échelle à partir duquel la clause (9a) est activée selon la première clause du PDI et la généralisation (12a). En Kabyle, toutes les géminées se voient systématiquement attribuées le trait [- continu] de l'alternance. La condition «environnement dérivé » est motivé uniquement par les segments stridents parmi les consonnes impliquées dans les dérivations morphologiques. Il n'a aucun effet sur les segments non-stridents puisque les géminées lexicales de ce type sont toujours [continu]. Cela veut dire que $/ \beta \beta /, / \theta \theta /$ et /ðð/ ne sont jamais attestés au niveau de la représentation lexicale du kabyle, contrairement aux géminées stridentes qui sont largement répandues. Mais la règle (9) ne rend pas compte du cas de l'uvulaire / $\chi /$ qui est une fricative non stridente mais qui ne participe pas à l'alternance fricative/occlusive géminée. Elmedlaoui n'a pas fourni d'explication pour rendre compte du comportement particulier de cette obstruante mais a signalé que toutes les formes qu'il a pu trouver sont des emprunts à l'arabe. Le cas des uvulaires est effectivement un cas assez particulier qui nécessite une étude approfondie pour déterminer exactement leurs particularités phonétiques et leurs comportements phonologiques. Le cas des uvulaires sera traité brièvement plus bas. L'analyse d'Elmedlaoui (1993), contrairement aux approches de Hayes et de Schein et Steriade, et en suivant l'approche initiée par Churma, fait le lien entre l'inaltérabilité et le phénomène de la lénition (voir aussi Kirchner 2000). La même approche sera adoptée ici, tout en postulant pour le $\mathrm{PCH}$, un processus de spirantisation au lieu d'occlusivication.

\footnotetext{
${ }^{5}$ Les traits de fortition sont : - vocoide, - approximante, - sonorante, + consonantique, + - bas, - continu, +
} 


\section{La spirantisation en berbère chleuh}

Les berbérisants du $19^{\mathrm{e}}$ et du début du $20^{\mathrm{e}}$ siècles se sont basés sur des considérations géographiques et linguistiques, phonétiques plus particulièrement, pour classer les parlers berbères. Au Maroc, cette classification distingue entre trois grandes aires dialectales :

1. Le rifain dans le Nord et le Nord-Est ;

2. Le tamazight dans le Centre et le Sud-Est ;

3. Le chleuh dans le Sud et le Sud-Ouest.

La spirantisation des occlusives, entre autres, a servi de facteurs discriminant pour élaborer cette classification. Biarnay (1917), par exemple, considère la spirantisation des occlusives comme un des plus importants traits qui permettent de distinguer les parlers du Nord des parlers chleuhs. Le tamazight et le rifain sont considérés comme des parlers spirants et le chleuh comme parler occlusif. Saïb (1974 : 2) va dans le même sens : «The southern dialects (e.g. Tachelhit and Twareg) lack the series of non-strident spirants and exhibit instead simple stops in cognate alternations. ». Les parlers chleuhs ne sont pas tous des parlers occlusifs. Le $\mathrm{PCH}$, qui est un parler chleuh parlé dans le Haut-Atlas, spirantise la labiale sonore ainsi que les valaires et les labiovélaires. Galand l'avait déjà signalé en (1953: 226) : «J'ai déjà rappelé que «les occlusives » ne sont pas parfaites dans tous les parlers du groupe chleuh»

Seules la labiale sonore $/ \mathrm{b} /$, les vélaires $/ \mathrm{k}$, g/ et les labiovélaires $/ \mathrm{k}^{\circ}, \mathrm{g}^{\circ} /$ se spirantisent en PCH. Les dentales ne spirantisent pas. Selon Fergusson : «Across-the-board spirantisation processes tend to start with labials and velars and then spread to dentals » (Cité par El Kirat 1987 : 195). Une preuve de cette tendance générale est que généralement seuls les parlers qui atteignent différents degrés de spirantisation des vélaires $\left(\mathrm{x}, \mathrm{\gamma}, \int, 3, \varnothing\right)$ spirantisent aussi les dentales (voir le cas des parlers berbères résumés dans Ridouane 1999). D’après une base de données établie par Lavoie (1996), un examen d'une liste de 27 langues où la spirantisation opère a été établi. Les résultats présentés dans un travail antérieur (Ridouane 1999) montrent que seule une langue (Dahalo, parlée au Kenya) spirantise les dentales mais pas les vélaires. Toutes les autres langues qui spirantisent les dentales spirantisent aussi les labiales et les vélaires. A l'inverse, les langues qui spirantisent les labiales et les vélaires ne spirantisent pas forcément les dentales.

distributed, + spread glottis, + stiff vocal cords. 


\subsection{Le statut phonologique des spirantes}

Le PCH présente une alternance entre spirantes simples et occlusives géminées.

$\begin{array}{lll}\text { Aoriste } & \underline{\text { Inaccompli }} & \\ \mathrm{r} \beta \mathrm{u} & \text { rbbu } & \text { «porter» } \\ \mathrm{xl} & \mathrm{kkal} & \text { «passer la nuit } \\ \mathrm{\gamma n} & \text { ggan } & \text { «dormir» }\end{array}$

Pour rendre compte de l'alternance spirante simple occlusive géminée illustrée ci-dessus, on a à priori le choix entre deux règles: une règle d'occlusivication ou une règle de spirantisation, selon le statut phonologique attribué aux spirantes. L'analyse défendue ici est qu'il s'agit d'une règle de spirantisation qui modifie les occlusives non coronales simples en spirantes correspondantes.

L'alternance présentée dans (13) est attestée dans plusieurs parlers berbères du Nord (e.g. le parler rifain d'Ayt Ndhir (Saïb 1976), le parler rifain de Beni Iznassen (El Kirat 1987) et le kabyle (Chaker 1984)). Coome cela a été signalé auparavant, différents auteurs considèrent que la direction historique du changement occlusive / spirante ne peut plus rendre compte des données synchroniques, et postulent à l'inverse une règle d'occlusivication spirante $\rightarrow$ occlusive. Les raisons que ces auteurs ont avancées pour justifier cette approche ne s'appliquent pas au cas du PCH. Dans le parler rifain de Beni Iznassen (PBI), décrit par El Kirat, les coronales simples /t, d/ se réalisent / $\theta$, ð/, sauf quand elles sont immédiatement au contact de /m, n, 1/, auquel cas les coronales simples restent occlusives. Les vélaires et leurs homologues labialisées se spirantisent dans tous les contextes. La gémination, comme en $\mathrm{PCH}$, bloque la spirantisation. Un ensemble d'arguments justifie selon El Kirat le statut phonémique des spirantes. Premièrement, ces segments sont attestés dans la forme verbale de base, qui est la catégorie la moins marquée. Deuxièmement, le parler rifain contient une grande quantité d'emprunts arabes qui sont intégrés au lexique local tout en gardant l'aspect occlusif de leurs segments. Cette adaptation des occlusives sans les spirantiser a été facilitée par la réduction quantitative qui a affecté les géminées, comme le montrent les formes (14). Les mots de la deuxième colonne sont leurs contreparties en $\mathrm{PCH}$ où la gémination est maintenue : 


\begin{tabular}{|c|c|c|}
\hline PBI & $\mathrm{PCH}$ & \\
\hline Өabrat & taßratt & «lettre» \\
\hline$\theta$ azðat & tusditt & «femme maigre» \\
\hline$\theta$ azəlit & taylitt & «reine » \\
\hline
\end{tabular}

Troisièmement, et c'est là l'argument majeur selon El Kirat, la spirantisation n'est plus un processus synchroniquement productif. La même tendance a été observée en kabyle (Chaker 1984). La situation actuelle dans ce dernier parler se caractérise par un enrichissement du système phonémique par des occlusives qui ne participent plus à l'alternance spirante/occlusive. L'émergence de cette nouvelle série d'occlusives simples fait dire à Chaker (ibid : 85) «[...] pour toutes ces occlusives simples, on assiste incontestablement à une évolution générale allant dans le sens de leur phonologisation. » $\mathrm{Au}$ vu de cette nouvelle situation, Elmedloui (1993 : 160) conclut : «This new situation makes it impossible to state a spirantizing rule for Rifain and Kabyle, since only segments of the ancient lexical stratum participate in the lexical distribution or morphological and phonological alternation, between simplex continuants and geminates stops.»

La situation en PCH est différente, dans le sens où un ensemble d'arguments indique plutôt que les spirantes dérivent d'occlusives sous-jacentes. Un premier argument est lié à la tendance universelle des langues à préférer les occlusives aux fricatives dans la structure sousjacente. Un système phonologique avec une occlusive / $/ \mathrm{k} /$ est plus naturel qu'un système contenant une spirante /x/. L'existence de /x/ présuppose l'existence de sa contrepartie occlusive / $\mathrm{k} /$ et non pas l'inverse. D'après Jakobson (1968: 51): «The acquisition of fricatives presupposes the acquisition of stops in child language; and in linguistic systems of the world the former cannot exist unless the latter exists.»

Un deuxième argument important est que les géminées occlusives labiales et vélaires en $\mathrm{PCH}$ restent géminées et se distinguent des simples spirantes aussi bien en terme de durée qu'en terme de mode d'articulation (cf. Loauli et Maddieson 1999, Ridouane 2003). Ceci n'est pas le cas pour les occlusives géminées en rifain ou en kabyle qui, contrairement au PCH, ont tendance à se dégéminer et à se réaliser comme des occlusives simples, le mode d'articulation occlusive $v s$. fricative étant suffisant pour les distinguer des spirantes. Le maintien de l'aspect géminé des occlusives en $\mathrm{PCH}$, qui est apparemment contraire au principe d'économie, 
s'explique essentiellement par l'existence d'une large série d'occlusives simples non spirantisées : les dentales /t, d/ et les emphatiques /T, D/. Cet aspect distingue encore d'avantage le $\mathrm{PCH}$ des parlers berbères du Nord où la spirantisation a affecté tous les lieux d'articulation. Ce maintien de la gémination s'explique aussi par l'existence en chleuh de la série occlusive ([b], $[\mathrm{k}]$ et $[\mathrm{g}]$ ) qui est facilement identifiée et réalisée par les locuteurs du PCH. Certains locuteurs peuvent en effet supprimer la spirantisation en parlant avec les locuteurs d'un autre parler chleuh non spirant. C'est le cas personnel de l'auteur de ce travail, qui, en tant que locuteur natif du $\mathrm{PCH}$, spirantise généralement en parlant avec les membres de sa famille ou avec tout autre locuteur natif du $\mathrm{PCH}$, mais rarement avec les autres Chleuhs. Les locuteurs du PCH considèrent les formes /akal/ et /axal/ comme deux prononciations d'un même mot qui signifie "terre". L'unique caractéristique distinctive de ces deux formes est de nature sociophonétique. En effet, il suffit qu'un locuteur d'un autre parler chleuh entende un locuteur du PCH pour remarquer l'existence de cette variante et si on lui pose la question il croit savoir d'où vient la personne qui a parlé.

Pour finir, un aspect important distingue le PCH des parlers du Nord : la spirantisation est un processus synchronique en PCH. En effet, les locuteurs de ce parler spirantisent les occlusives des mots empruntés aux autres langues, en l'occurrence l'arabe marocain, comme le montrent les formes (15). Les formes dans la troisième colonne sont leurs contreparties dans le parler rifain de Beni Iznassen (voir El Kirat 1987). Notez la différence dans la nature des vélaires entre ces deux parlers berbères :

$\begin{array}{llll}\text { PCH } & \text { Arabe marocain } & \text { PBI } & \\ \text { lxas } & \text { lkas } & \text { lkas } & \text { « verre » } \\ \hbar x m & \hbar k e m & \hbar k e m & \text { «gouverne » } \\ \text { lxttan } & \text { lkettan } & \text { lkettan } & \text { «tissu » } \\ \text { ayzzar } & \text { lgezzar } & \text { agezzar } & \text { «boucher }\end{array}$

\section{Les géminées et la spirantisation}

Il y a plusieurs types de géminées en berbère chleuh : des géminées lexicales, morphologiques et phonologiques. Les géminées lexicales, dont les occurrences sont imprédictibles, doivent être représentées dans le lexique. Des exemples illustrant cette opposition sont présentés cidessous : 


$\begin{array}{llll}\text { tut } & \text { «elle a oublié » } & \text { ttut } & \text { « oublie-le » } \\ \text { ifis } & \text { «loup » } & \text { ifiss } & \text { «il est silencieux » } \\ \text { imi } & \text { «bouche » } & \text { immi } & \text { «ma grand-mère » } \\ \text { inas } & \text { « dis lui » } & \text { innas } & \text { «sa mère » } \\ \text { ili } & \text { « aie, imp. » } & \text { illi } & \text { «ma fille » }\end{array}$

Les géminées morphologiques dérivent de processus morphologiques comme la formation des inaccomplis (17a) ou la dérivation des pluriels (17b) :

\begin{tabular}{|c|c|c|}
\hline Aoriste & \multicolumn{2}{|l|}{$\underline{\text { Inaccompli }}$} \\
\hline ls & lssa & «porter $»$ \\
\hline $\mathrm{ftu}$ & $\mathrm{fttu}$ & «marcher » \\
\hline sR & ssaR & «acheter» \\
\hline$\underline{\text { Singulier }}$ & $\underline{\text { Pluriel }}$ & \\
\hline afus & ifassn & «main» \\
\hline aDar & iDarrn & «pied » \\
\hline
\end{tabular}

Les géminées phonologiques dérivent d'une assimilation entre deux segments adjacents. Cidessous quelques exemples où les suffixes du féminin ou de la forme diminutive, sont attachés à des mots se terminant par des consonnes partageant le même lieu d'articulation :

$$
\begin{array}{lll}
/ \mathrm{t}-\mathrm{afud}-\mathrm{t} / & \text { tafutt } & \text { «genou } \\
/ \mathrm{t}-\mathrm{agDiD}-\mathrm{t} / & \text { tagDiTT } & \text { « oiseau } ~
\end{array}
$$

Un dernier exemple concerne les géminées hétéromorphémiques dérivées par concaténation : (e.g. yiwid\#dis « il a ramené avec lui »). Quand deux segments identiques sont séparés par une frontière morphologique, à l'instar de ce qui se produit dans plusieurs langues, une géminée est dérivée qui se produit avec une même durée que les géminées tautomorphémiques (voir Ouakrim 1994, Ridouane 2003).

Ces différents types de géminées ne réagissent pas tous de la même manière vis-à-vis de la spirantisation. Les géminées lexicales, et c'est là une tendance universelle, ne se spirantisent jamais. Les géminées issues d'une assimilation complète résistent aussi à l'application de cette règle. Par contre, les géminées issues d'une concaténation se spirantisent. Considérons les formes suivantes : 

a. likkis/ $\rightarrow$ ikkis $\rightarrow$ poignée »
$\begin{array}{llll}\text { /tukkimt/ } & \rightarrow & \text { tukkimt } & \text { «poignée » } \\ \text { b. } \quad \text { liwik kiyyi/ } & \rightarrow & \text { iwixxiyyi } & \text { «ton fils à toi » } \\ \text { /innayak kra/ } & \rightarrow & \text { innayaxxra } & \text { «il t'a dit quelque chose» }\end{array}$

Le fait que les géminées hétéromorphémiques (19b) présentent des différences au niveau phonologique implique que leur représentation doit être distincte de celle des géminées tautomorphémiques (19a). Comme indiqué plus haut, la représentation Autosegmentale permet une telle différenciation et peut donc, comme pour le tigrinya, rendre adéquatement compte de la spirantisation des géminées hétéromorphémiques. Les deux représentations cidessous reflètent cette différence :

a. Inaltérabilité des géminées

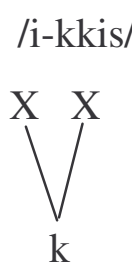

*ixxis b. Spirantisation de /k\#k/

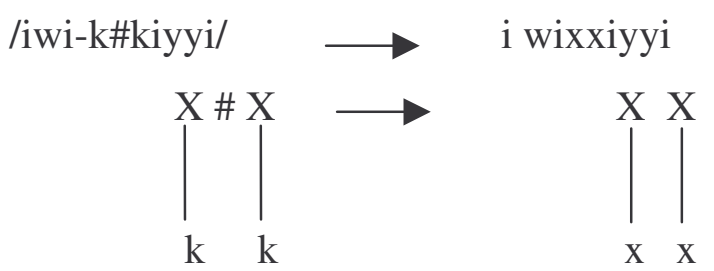

Considérons maintenant le cas des différences potentielles entre les géminées par concaténation et les géminées issues d'une assimilation complète. Cet aspect est illustré en se servant du comportement des pronoms d'objet direct $/ \mathrm{k} /$ et $/ \mathrm{k}^{\circ} \mathrm{n} /$ qui marquent la deuxième personne du singulier et du pluriel, respectivement. Quand ces pronoms d'objet directe précèdent des verbes qui se terminent par une vélaire comme dans (21) ci-dessous, la spirantisation opère, donnant ainsi lieu à des spirantes $/ \mathrm{xx} /$ et $/ \mathrm{xx}^{\circ} /$ longues :

$$
\begin{array}{lll}
/ \mathrm{t}-\mathrm{s}-\hbar \mathrm{rrk}=\mathrm{k} / & \operatorname{tsh} \mathrm{rrxx} & \text { 《elle t'a fait bouger » } \\
/ \mathrm{t}-\hbar \mathrm{rg}=\mathrm{k}^{\circ} \mathrm{n} / & \operatorname{thrxx}^{\circ} \mathrm{n}^{6} \quad \text { 《elle vous a brûlés » }
\end{array}
$$

Considérons maintenant le comportement de ces mêmes pronoms d'objet direct quand ils précèdent le verbe :

$$
\begin{aligned}
& \text { /rad=k i-hrg/ rakk iћrg "il te brûlera" } \\
& / \mathrm{rad}=\mathrm{k}^{\circ} \mathrm{n} \mathrm{i}-\mathrm{hrg} / \quad \operatorname{rakk}^{\circ} \mathrm{n} \mathrm{i} h \mathrm{rg} \quad \text { "il vous brûlera" }
\end{aligned}
$$

\footnotetext{
${ }^{6} \mathrm{Le} \mathrm{PCH}$ a une règle de dévoisement régressif qui dévoise toute fricative, sous-jacente ou résultant de la spirantisation, devant une sourde (voir Ridouane 2003).
} 
Les géminées dans (22) sont issues d'une assimilation complète de la consonne /d/. Il résulte de cette assimilation une association du nœud racine de la consonne initiale du prénom d'objet direct $/ \mathrm{k} /$ à la position $\mathrm{X}$ de la dernière consonne de la forme rad (voir Halle \& Vergnaud 1980, Steriade 1982).. Le /d/ sous jacent s'efface par convention « Stray Erasure» (cf. McCarthy 1981). Cette règle est formulée dans (23) :

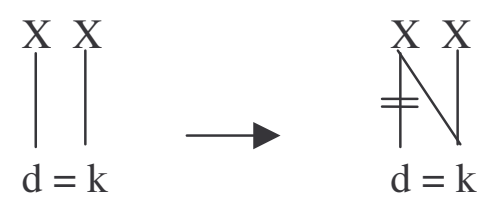

L'output de cette ré-association est une vélaire liée à deux positions dans le squelette. Cette vélaire a donc la même représentation que celle des géminées lexicales ce qui expliquerait, si l'on adopte l'approche notationnelle, leur comportement identique. Mais comme l'a souligné Elmedlaoui (voir plus haut), et Churma (1988) avant lui, de telles représentations ne disent rien sur les raisons de cette inaltérabilité. L'explication fournie par Churma (1988) stipule que c'est la force inhérente aux géminées qui empêcherait tout processus d'affaiblissement comme la spirantisation de les affecter. Mais une telle explication souffre d'un manque de données objectives pour la soutenir. Aucun argument émanant de données de phonétique expérimentale n'a pas par exemple été fourni en faveur d'une telle analyse. Il faudra en effet démontrer (1) que les géminées lexicales présentent des caractéristiques d'une articulation tendue, (2) que des différences existent au niveau phonétique entre les géminées lexicales et issues d'une assimilation complète d'un côté et les géminées par concaténation de l'autre, et (3) que la différence dans leur comportement vis-à-vis de la spirantisation est due à ces différences. Une telle analyse phonétique est fournie dans Ridouane (2006) et Ridouane (sous presse) (voir aussi Ouakrim 1994). Les résultats d'un ensemble d'analyses acoustiques et articulatoires montrent que la gémination lexicale est phonétiquement implémentée par différents corrélats, dont le principal est la durée. Les données obtenues par électropalatographie montrent que ces différences de durée valent même pour les occlusives sourdes après pause. Cette distinction temporelle est renforcée par des corrélats secondaires comme l'abrègement de la voyelle précédente, l'amplitude du relâchement, le dévoisement des géminées occlusives, le maintien systématique d'une occlusion complète, etc.. Ces corrélats sont interprétés comme des manifestations de la force articulatoire qui caractérise ces segments. Concernant la comparaison entre les différents types de géminées, les résultats montrent que les trois types de segments présentent pratiquement les mêmes durées (ce qui soutiendrait leur représentation identique au niveau du palier prosodique, si l'on interprète ces 
positions comme des unités de durée). Par contre des différences notables ont été observées entre les géminées lexicales et issues d'une assimilation complète d'un côté et les géminées par concaténation de l'autre. Tandis que les géminées par assimilation, comme les géminées lexicales, sont renforcées par les attributs acoustiques de la force articulatoire (abrègement de la voyelle, amplitude du relâchement, etc.), les géminées par concaténation ne le sont pas. Il y a donc lieu de distinguer entre les vraies géminées (lexicales et issues d'une assimilation complète) phonétiquement implémentées par des corrélats secondaires responsables de leur aspect tendu et les fausses géminées (géminées par concaténation) qui résultent uniquement de l'adjacence entre deux segments identiques. Sur la base de ces résultats, on peut donc légitimement postuler en accord avec Churma (1988: 1) que «... it is the «inherent» strength of geminate consonants [...] that prevents any (non-assimilatory) weakening process other than degemination from affecting them ». Une lien peut être établi entre cette observation et une propriété - plutôt inattendue - que manifeste les corrélats de renforcement. En se basant sur plusieurs exemples tirés de différentes langues, Keyser \& Stevens (2006) ont observé que tandis que les corrélats primaires sont, dans certains contextes, sujets à des processus d'affaiblissement (voir d'élision), les traits de renforcements sont beaucoup plus robustes et ne sont apparemment jamais affaiblis ou effacés. En PCH, les corrélats de renforcement des géminées, ceux responsables de l'aspect tendu affiché par ces segments, résistent aussi à l'application de règles d'affaiblissement. Autrement dit, une fois introduits pour renforcer une opposition entre deux segments, les corrélats de renforcement tendent de survivre et peuvent même supplanter le corrélat de base qu'ils sont censés à l'origine renforcer.

\section{Le cas des géminées morphologiques : quelques réflexions...}

Les géminées morphologiques, comme nous l'avons déjà signalé, présentent une alternance entre spirantes simples et occlusives géminées. D'autres types d'alternance que ceux cités dans (13) sont aussi attestés en PCH (et d'autres variétés du chleuh) :
a. /D/ $\approx \quad / \mathrm{tt} /$
$\mathrm{Di}$ TTay
"virer"
b. $/ \mathrm{R} /$

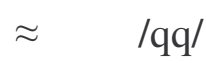
$\mathrm{rR}$
rqqa
"se réchauffer" 
Ces alternances ont fait l'objet de plusieurs études (Elmedlaoui 1985, Lasri 1991). Elmedlaoui a ainsi décrit le même phénomène dans le parler chleuh d'Imdlawn (PCI). Comme en PCH, cette alternance morphologique caractérise le processus de la formation de l'inaccompli :

$\begin{array}{llll} & \underline{\text { Aorsite }} & \underline{\text { Inaccompli }} & \\ \text { a. } & \operatorname{Rr} & \text { aqqra } & \text { «lire } \\ \text { b. } & \mathrm{kDu} & \mathrm{kTTu} & \text { « sentir» } \\ \text { c. } & \mathrm{rwi} & \operatorname{rgg}^{\circ} \mathrm{i} & \text { «mélanger }\end{array}$

L'analyse d'Elmedlauoi, qui ne concerne que les alternances $[\mathrm{R}] /[\mathrm{qq}]$ et $[\mathrm{w}] /\left[\mathrm{gg}^{\circ}\right]$, est résumée dans les points suivants :

1. Il y a une opposition sous-jacente /R/ vs. /q/ et /w/ vs. /g\%;

2. [w] peut apparaître en surface ;

3. Il n'y a jamais d'alternance $\left[\mathrm{g}^{\circ}\right] /\left[\mathrm{gg}^{\circ}\right]$ ou $[\mathrm{w}] /[\mathrm{ww}]$;

4. La gémination morphologiquement alternante présente le phénomène d'occlusification.

Le parler chleuh de Tiznit (PCT), décrit par Lasri (1991), contient aussi les mêmes géminées à mutation que celles du PCI (43). Lasri, dont l'analyse est principalement développée dans le cadre de la Phonologie Lexicale, part des constatations suivantes :

1. Hormis quelques exceptions, les géminées /RR/, /DD/ et /ww/ tautomorphémiques sont interdites ;

2. Dans les cas où la gémination est produite par un processus morphologique, les instances /RR/, /DD/ et /ww/ sont réalisées respectivement, /qq/, /TT/ et /gg / ;

3. Au niveau lexical, les géminées /RR/, /DD/ et /ww/ sont possibles mais seulement comme résultat d'une assimilation.

En partant du principe de la préservation des structures, l'auteur postule que les géminées /RR/, /DD/ et /ww/, étant interdites dans les structures lexicales du PCT, sont tout naturellement prohibées aux autres stades de formation des mots. Au niveau post-lexical, le principe de préservation des structures n'est plus en vigueur. Ceci explique donc, selon lui, les occurrences des géminées [RR], [DD] et [ww] dans (26) produites par assimilation de deux segments qui ne font pas partie d'un même morphème : 


$$
\begin{array}{llc}
\text { /t-Duf/ } & \text { DDuf } & \text { « elle surveille» } \\
\text { / rad nR-R/ } & \text { radnRR } & \text { «je vais tuer» } \\
\text { /yan ass/ } & \text { yawwas } & \text { « un jour » }
\end{array}
$$

L'analyse défendue par Lasri pour expliquer ces alternances est résumée ci-dessous :

1. L'interdiction des géminées /RR/, /DD/ et /ww/, qui sont les trois qui ne figurent pas au niveau lexical ;

2. Les traits mis en œuvre afin de réaliser les correspondances sont les traits /voisé/ et /continu/. Dans les trois cas, ce sont les valeurs de ces deux traits qui sont affectées ;

3. Les traits de lieu d'articulation n'entrent pas en ligne de compte (i.e. dorsal et pharyngal pour R/qq, labial pour ww/gg ${ }^{\circ}$, coronal et pharyngal pour D/TT).

Les faits qui sous-tendent l'analyse de Lasri ainsi que celle d'Elmedlaoui ne peuvent pas être valides pour le $\mathrm{PCH}$, car les faits ne sont pas les mêmes. La géminée /ww/ existe bel et bien au niveau lexical en $\mathrm{PCH}$ :

$\begin{array}{ll}\text { anuwwaS } & \text { «mouchard } \\ \text { tawwuri } & \text { «travail } \\ \text { tawwuxt } & \text { «hibou }\end{array}$

Dans le cas où la gémination est produite par un processus morphologique, /w/ ne se réalise que par sa correspondante géminée identique $[\mathrm{ww}]$ et non $\left[\mathrm{gg}^{\circ}\right]$ :

$\begin{array}{lll}\text { nwu } & \text { nwwu } & \text { «cuire » } \\ & * \operatorname{ngg}^{\circ} \mathrm{i} & \\ \text { rwi } & \text { rwwi } & \text { «mélanger » } \\ & * \operatorname{rgg}^{\circ} \mathrm{i} & \\ \mathrm{zwu} & \mathrm{zwwu} & \text { « sécher » } \\ & * \mathrm{zgg}^{\circ} \mathrm{u} & \end{array}$

Enfin, /w/ et /g\% ainsi que /ww/ et $/ \mathrm{gg}^{\circ} /$ sont en opposition :

$\begin{array}{llll}\text { awi } & \text { «prend, imp » } & \mathrm{ag}^{\circ} \mathrm{i} & \text { «refuse, imp » } \\ \text { iwza } & \text { «il a dépecé » } & \operatorname{ig}^{\circ} \mathrm{za} & \text { « il est dégoûtant » } \\ \text { tawwuxt } & \text { «hibou » } & \operatorname{tagg}^{\circ} \mathrm{uxt} & \text { « tu es loin » }\end{array}$


Les même remarques s'appliquent aussi aux cas des dentales emphatiques. /DD/ existe au niveau lexical :

(30)

$$
\begin{aligned}
& \text { tiDDa } \\
& \text { taDDinga } \\
& \text { iDDr }
\end{aligned}
$$

$$
\begin{aligned}
& \text { « sangsue » } \\
& \text { « vague » } \\
& \text { «il est tombé » }
\end{aligned}
$$

Dans le cas où la gémination est produite suite à un processus morphologique, la dentale

\begin{tabular}{|c|c|c|}
\hline $\mathrm{bDu}$ & bDDu & «partager $»$ \\
\hline & *bTTu & \\
\hline $\mathrm{xDu}$ & $\mathrm{xDDu}$ & « sentir » \\
\hline & *xTTu & \\
\hline$\hbar \mathrm{Du}$ & $\hbar \mathrm{DDu}$ & « cacher » \\
\hline & $* \hbar \mathrm{TTu}$ & \\
\hline
\end{tabular}
emphatique peut se réaliser de trois façons différentes :

a. [DD] ou [TT]

$\begin{array}{lll}\mathrm{rDl} & \mathrm{rDDl} & \\ \mathrm{nDr} & \mathrm{rTTL} & \\ & \mathrm{nDDr} & \text { «sauter } » \\ & \mathrm{nTTr} & \end{array}$

b. [DD]

c. $[\mathrm{TT}]$

$\begin{array}{lll}\text { Dr } & \text { TTar } & \text { «tomber } ~ \\ \text { Di } & \text { TTay } & \text { « virer }\end{array}$

La gémination de /D/ peut donc se réaliser aussi bien comme /TT/ que comme $/ \mathrm{DD} /{ }^{7}$. Certains exemples attestent même de l'existence d'une opposition entre ces deux géminées :

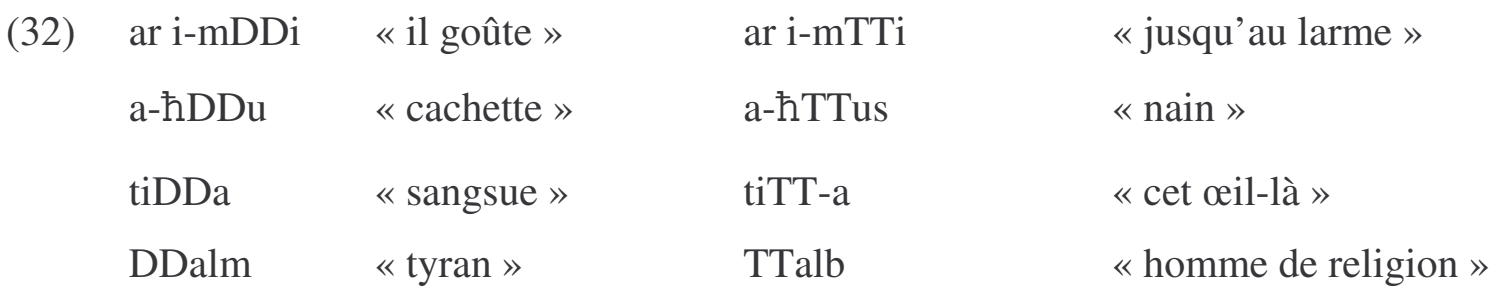

\footnotetext{
${ }^{7}$ Rien ne semble motiver l'une ou l'autre réalisation. La seule différence observable est que les formes réalisées uniquement avec /TT/ contiennent toutes le segment simple en position initiale du verbe.
} 
Nous ne comptons pas développer les faits des deux cas ci-dessus, l'objectif est de souligner qu'une analyse de la gémination à mutation en PCH ne peut pas aligner l'alternance R/qq sur celle de D/TT ou w/gg ${ }^{\circ}$, comme c'est le cas dans Elmedlaoui (1985) et Lasri (1991). Une alternative, qui semble intéressante à examiner de plus près, serait plutôt d'aligner l'alternance R/qq sur l'alternance spirante simple/occlusive géminée attestée dans plusieurs parlers berbères. L'argumentation est basée aussi bien sur les résultats des analyses phonétiques présentées dans Ridouane (2003) que sur la distribution de ces segments en PCH.

Les analyses acoustiques présentées dans Ridouane (2003) ont montré que /q/ et /qq/, contrairement aux autres occlusives simples et géminées, ne se distinguent pas en terme de durée d'occlusion. Les deux étant produites avec quasiment les mêmes durées. Nous avons aussi observé qu'aussi bien la simple /q/ que la géminée /qq/, à l'image des consonnes géminées, abrège la durée de voyelle précédente. Pour les autres occlusives, la voyelle ne s'abrège que devant la série géminée. Au niveau articulatoire nous avons aussi observé que la simple /q/ est produite avec une ouverture glottale systématiquement plus importante que les autres occlusives sourdes simples. Nous n'avons pas non plus observé de différences importantes entre la durée ou le degré d'ouverture glottale entre la simple /q/ et la géminée /qq/. A l'inverse, pour les autres occlusives, la géminée sourde est systématiquement produite avec une amplitude et une durée plus importantes que les simples correspondantes. Nous avons conclu au vu de ces résultats que l'uvulaire occlusive sourde se réalise principalement comme une géminée en PCH.

Les données sur la distribution de ce segment ainsi que de sa contrepartie fricative sonore confirment cette analyse. Les uvulaires attestés dans le PCH sont les sourdes [qq] et les sonores $[R]$ et $[R R]$ et leurs homologues labialisées. Les quatre seules formes où nous avons relevé la présence d'une uvulaire qui paraît intuitivement être réalisée comme une simple sont listées dans (33a) :

\begin{tabular}{|c|c|c|}
\hline a. & tasqaqqayt & « hoquet » \\
\hline & tiqqrqaw $\int$ in & « castagnettes » \\
\hline & ћqqiq & « regarder fixement » \\
\hline & tqssf & «il a rétréci » \\
\hline & i qqa & «il est difficile » \\
\hline
\end{tabular}




\begin{tabular}{|c|c|c|}
\hline & « ferraille » \\
\hline \multirow{2}{*}{\multicolumn{2}{|c|}{$\begin{array}{l}\text { alqq }{ }^{\circ} \text { nayn } \\
\text { iqqama }\end{array}$}} & «lapin » \\
\hline & & «il est resté » \\
\hline \multirow[t]{3}{*}{ c. } & aRrda & « souris » \\
\hline & aRalim & « canne » \\
\hline & $\mathrm{aR}^{\circ} \mathrm{lal}$ & « escargot» \\
\hline \multirow[t]{2}{*}{ d. } & $\mathrm{aRRu}$ & « lait fermenté » \\
\hline & taRRat & «brebis » \\
\hline \multirow[t]{3}{*}{ e. } & $\mathrm{RR}^{\circ} \mathrm{id}$ & « ceux-ci » \\
\hline & RRid & «par ici » \\
\hline & sRR & «acheter, 1.p.s. aoriste » \\
\hline
\end{tabular}

Un certain nombre de remarques est à faire à propos des données (33). Mis à part les formes (33a), /q/ simple est pratiquement inexistant dans l'inventaire lexical du PCH. Les deux premières formes de (33a) sont des onomatopées et la troisième est un emprunt récent à l'arabe. Notez que toutes ces formes contiennent dans le même radical une autre uvulaire sourde géminée. Les exemples de /q/ simple dans Elmedlaoui (1985 : 140), qui sont en grande majorité des emprunts à l'arabe, se réalisent en $\mathrm{PCH}$ comme des géminées :

$\begin{array}{lll}P C I & P C H & \\ \text { aquwwad } & \text { aqquwwad } & \text { "proxénète » } \\ \text { aqlmun } & \text { aqqlmun } & \text { «capuchon » } \\ \mathrm{q}^{\circ} \mathrm{nD} & \mathrm{qq}^{\circ} \mathrm{nD} & \text { «désespère » }\end{array}$

Le cas de la dernière forme $/ \mathrm{q}^{\circ} \mathrm{nd} /$ est intéressant. Dell \& Elmedlaoui (1991) ont analysé les mécanismes qui gouvernent le passage de l'aoriste à l'inaccompli. Ce passage se fait entre autres en géminant une consonne de la base verbale. Ces deux auteurs se sont basés sur la structure syllabique du verbe dans le choix du segment candidat à la gémination. D'après leur analyse, on gémine le segment qui occupe la position d'attaque de la syllabe : " in a verb base $[\ldots]^{8}$ geminate that segment which is a syllable onset." (ibid : 86). Nous partageons totalement cette analyse qui prévoit correctement la gémination de la première consonne dans les verbes ci-dessous ${ }^{9}$ :

\footnotetext{
${ }^{8}$ Voir Dell et Elmedlaoui $(1991: 85)$ sur un ensemble de conditions que la base verbale doit satisfaire pour être sujet à la gémination.

${ }^{9}$ Voir Boukous (1987), Jebbour (1996) et Lahrouchi (2000) pour une analyse alternative.
} 


\begin{tabular}{|c|c|c|}
\hline Aoariste & Inaccompli & \\
\hline xrf & kkrf & «attacher» \\
\hline frd & ffrd & «manger» \\
\hline $\mathrm{x}^{\circ} \mathrm{mz}$ & $\mathrm{kk}^{\circ} \mathrm{mz}$ & «se gratter $»$ \\
\hline
\end{tabular}

Les verbes à l'aoriste sont monosyllabiques dont la première consonne occupe la position d'attaque ; elle est par conséquent géminée à l'inaccompli. La structure syllabique de ces formes serait identique à celle de $/ \mathrm{q}^{\circ} \mathrm{nD} /$ si on suppose que le segment initial était une consonne simple. On devrait donc, pour son passage à l'inaccompli, géminer la première consonne: *[qq $\left.{ }^{\circ} \mathrm{nd}\right]$, or ce n'est pas le cas en $\mathrm{PCH}$, ni dans les autres variétés du chleuh d'ailleurs. La seule manière correcte de former l'inaccompli de ce verbe est de préfixer la géminée /tt/ à la base verbale : /ttqq ${ }^{\circ} \mathrm{naD} /$. Ce mécanisme de dérivation est identique à celui observé dans les verbes contenant une géminée à l'initiale. Pour ce type de consonnes, le passage de l'aoriste à l'inaccompli s'effectue justement en préfixant la géminée /tt/ :

$\begin{array}{lll}\text { Aoariste } & \underline{\text { Inaccompli }} & \\ \text { mmrz } & \text { ttmmraz } & \text { «frapper à la tête» } \\ \text { kks } & \text { ttkks } & \text { «enlever » } \\ \text { bbi } & \text { ttbbi } & \text { «déchirer » }\end{array}$

Considérons maintenant le cas des uvulaires fricatives sonores géminées. Mais avant de traiter de leurs distributions, rappelons brièvement les résultats obtenus dans la partie acoustique présentée dans Ridouane (2003). La différence de durée observée entre uvulaire simple/géminée, contrairement aux autres points d'articulation, n'est que très légèrement significative pour certains locuteurs, qui produisent une différence moyenne entre la simple et la géminée de $33 \mathrm{~ms}$ au lieu de $78 \mathrm{~ms}$ pour les autres fricatives. C'est la géminée /RR/ qui est généralement réalisée avec une durée moins importante que les autres fricatives.

Les uvulaires géminées dans (33d) sont les seules que nous avons pu relever. Boukous (1994) a déjà signalé que la distribution de ce segment est très limitée. Signalons que la forme /aRRu/ peut aussi être réalisée avec une uvulaire simple. Les géminées de (33e) sont de deux natures. $\left[R^{\circ} R^{\circ}\right]$ dans le premier exemple est issu d'une assimilation totale. Sa structure sous-jacente /R-wid/ « ceux-ci » peut avoir deux autres réalisations possibles en PCH : [Rwid] ou [wwid]. Ces deux réalisations sont en variation libre. Dans le deuxième exemple de (33e), la 
gémination provient d'une suite de deux consonnes identiques : /R-Rid/. La première uvulaire qui signifie « dans » se dévoise quand elle est immédiatement suivie d'une sourde :

$$
\begin{array}{lll}
\text { R-tid } & \chi \text { tid } & \text { «celles-ci } ~ \\
\text { R-tudrt } & \chi \text { tudrt } & \text { «dans la vie }
\end{array}
$$

La suite /R-R/ se réalise [RR] ou [RaR] lorsque /R/ situé en fin de verbe est immédiatement suivi du suffixe /R/ qui indique la première personne du singulier. Ainsi /rad sR-R/ peut se prononcer $[\mathrm{rad} \mathrm{sRR}]$ ou $[\mathrm{rad} \mathrm{sRaR}] ;[\mathrm{R}]$ et $[\mathrm{aR}]$ étant deux allophones propres au suffixe de la première personne du singulier en $\mathrm{PCH}^{10}$.

Les données phonétiques et phonologiques indiquent ainsi que /RR/ tautomorphémique et /q/ simple, hormis quelques rares exceptions, ne sont pas attestés en PCH. Seules existent la fricative simple /R/ et sa correspondante géminée /qq/. Dans le cas où la gémination est produite suite à un processus morphologique, /R/ alterne avec sa correspondante géminée /qq/. Une possibilité, qui nécessite à l'évidence un examen plus détaillé, serait ainsi d'aligner cette alternance sur celle qui caractérise les formes (13) sachant que dans tous ces cas on a une alternance fricative simple-occlusive géminée.

\footnotetext{
${ }^{10}$ Ce phénomène ne concerne pas uniquement les suites [R-R], ce suffixe a toujours ces deux réalisations en variation libre après consonne en $\mathrm{PCH}$, exemple : $[\mathrm{xrfR}]$ et $[\mathrm{xrfaR}]$ «'ai attaché ».
} 


\section{RÉFÉRENCES}

Barkai, M.A.R. (1974). On duration and spirantization in Biblical Hebrew. Linguistic Inquiry $5,456-459$.

Biarnay, S. (1917). Étude sur les dialectes berbères du Rif. Paris : Ernest Leroux.

Boukous, M. (1987). Phonotactique et domaine prosodique en berbère (parler tachelhit d'Agadir, Maroc). Thèse de Doctorat, Université Paris 8.

Boukous, M. (1994). Variation phonique et compétence globale: le cas du parler d'Agadir. Publications de la Faculté des Lettres. Rabat.

Chaker, S. (1984). Textes en linguistique berbère. Paris : Editions du CNRS.

Churma, D. (1988). On 'on geminates'. Ms., Suny-Buffalo.

Dell, F. \& Elmedlaoui, M. (1991). Clitic ordering, morphology and phonology in the verbal complex of Imdalwn Tashlhiyt Berber. Part II: Langues Orientales Anciennes Philologie et Linguistique 3, 77-104.

El Kirat, Y. (1987) Spirantization in the Beni Iznassen dialect, diachrony and synchrony. Mémoire de D.E.S.S. Université Mohammed V, Rabat.

Elmedlaoui, M. (1985). Le parler berbère chleuh d'Imdlawn (Maroc); segments et syllabation. Thèse de Doctorat, Université de Paris 8.

Elmedlaoui, M. (1993). Gemination and spirantization in Hebrew, Berber, and Tigrinya: a fortis-Lenis module analysis. Linguistica Communicatio V, 1, 121-176.

Foley, J. (1977). Foundations of phonological theory. Cambridge University Press

Galand, L. (1953). La phonétique en dialectologie berbère. Orbis II/1, 225-233. 
Halle, M. \& Vergnaud, J.R. (1980). Three-dimensional Phonology. Journal of Linguistic Research 1, 83-105.

Hayes, B. (1986). Inalterability in CV phonology. Language 62, 321-351.

Inkelas, S. \& Cho, Y. (1991). Inalterability as prespecification. Language 69, 529-574.

Jakobson, R. (1968). Child language, aphasia, and phonologic universals. Mouton, The Hague.

Jebbour, A. (1996). Morphologie et contraintes prosodiques en berbère (tachehit de tiznit). Analyse linguistique et traitement automatique. Doctorat d'Etat. Université Mohamed V, Rabat.

Karlsson, F. (1983). Finnish grammar. Werner Söderström, Porvoo.

Keyser, J. \& Stevens, K. (2006). Enhancement and overlap in the speech chain. Language 82.1, 33-63.

Kirchner, R. (2000). Geminate inalterability and lenition. Language 76(3), 509-545.

Lahrouchi, M. (2001). Aspects morphophonologiques de la dérivation verbale en berbère (parler chleuh d'Agadir). Thèse de Doctorat, Université Paris 7.

Lasri, A. (1991). Aspects de la phonologie non-linéaire du parler berbère chleuh de Tidli. Thèse de Doctorat, Université Paris 3.

Lavoie, L. (1996). Consonant strength: results of a data bas development project. Working Papers of the Cornell Phonetics Laboratory 11, 269-316.

Louali, N. \& Maddieson, I. (1999). Phonological contrast and phonetic realization : the case of Berber stops. Proceedings of the $14^{\text {th }}$ International Congress of Phonetic Sciences, 603606. 
McCarthy, J. (1981). A prosodic theory of nonconcatenative morphology. Linguistic Inquiry $12,373-418$.

Ouakrim, O. (1994). Un paramètre acoustique distinguant la gémination de la tension consonantique. Études et Documents Berbères 11, 197-203.

Ridouane, R. (1999). La spirantisation dans un parler berbère du Maroc (parler chleuh de Haha). Diplôme d'Etudes Approfondies, Université Paris 3.

Ridouane, R. (2003) Les suites consonantiques en berbère chleuh ; phonétique et phonologie. Thèse de Doctorat, Université Paris 3.

Ridouane, R. (2006). Gemination at the junction of phonetic and phonology. $10^{\text {th }}$ Conference on Laboratotory Phonology, Paris. Présentation orale.

Ridouane, R. (sous presse). Gemination in tashlhiyt Berber: Acoustic and Articulatory analysis. Journal of the International Phonetic Association.

Saïb, J. (1974). Gemination and spirantization in Berber : diachrony and synchrony. Studies in African Linguistics 5, 1-26.

Saïb, J. (1976). A phonological study of Tamazight Berber: Dialect of the Ayt Ndhir. Ph.D. Dissertation, University of California Los Angeles.

Schein, B. \& Steriade, D. (1986). On geminates. Linguistic Inquiry 17, 691-744

Selkirk, E. (1990). A two-root theory of length. University of Massachusetts Occasional Papers 14,123-171.

Steriade, D. (1982). Greek prosodies and the nature of syllabification. Ph.D. Dissertation, Cambridge, Mass.: MIT.

Tangi, O. (1991). Aspects de la phonologie d'un parler berbère du Maroc : Ath-Sidhar (Rif). Thèse de Doctorat Unifié, Université Paris 8. 
